\section{Uso de medicamentos entre idosos residentes em áreas urbanas e rurais de município no Sul do Brasil: um estudo de base populacional}

\author{
Use of medication by the elderly in urban and rural \\ areas in southern Brazil: a population-based study
}

\footnotetext{
1 Faculdade de Medicina, Universidade Federal do Rio Grande do Sul, Porto Alegre, Brasil.

2 Faculdade de Odontologia Universidade Federal do Rio Grande do Sul, Porto Alegre, Brasil.

3 Faculdade de Odontologia de Piracicaba, Universidade Estadual de Campinas, Piracicaba, Brasil.

Correspondência T. S. Dal Pizzol Programa de Pós-graduação em Epidemiologia, Faculdad de Medicina, Universidade Federal do Rio Grande do Sul. Rua Ramiro Barcelos 260, Porto Alegre, RS 90035-003, Brasil.

tatiane.silva@ufrgs.br
}

\begin{abstract}
The study aimed to measure use of medication and polypharmacy among the elderly in Carlos Barbosa, Rio Grande do Sul State, Brazil, and to compare socio-demographic, economic, and health characteristics in relation to area of residence (urban versus rural) in a random sample of 811 persons 60 year of age or older. Interviews were used to collect data on socio-demographic characteristics, chronic illnesses, and self-reported use of medications. The association between area of residence and medication or polypharmacy was adjusted for confounders using Poisson regression with robust variance. Prevalence rates for use of medication and polypharmacy were higher among older persons living in the urban area. Living in the urban area was positively and independently associated with use of medication $(P R=1.10$; 95\%CI: 1.02-1.20) and polypharmacy $(P R=1.83$; 95\%CI: 1.27-2.65) in this group of elderly in southern Brazil.
\end{abstract}

Aged; Drug Utilization; Health Services Accessibility; Rural Health
Tatiane da Silva Dal Pizzol 1

Emilia da Silva Pons 1

Fernando Neves Hugo 2

Mary Clarisse Bozzetti 1

Maria da Luz Rosário de Sousa ${ }^{3}$

Juliana Balbinot Hilgert 2

\section{Introdução}

O crescimento da população idosa representa um dos grandes desafios da saúde pública no Brasil. Estimativas indicam que a população idosa brasileira poderá exceder 30 milhões de pessoas até 2020, chegando a representar quase $13 \%$ da população 1 . A proporção de idosos vem crescendo mais rapidamente que a proporção de crianças, seguindo uma tendência mundial. Se em 1980 existiam 16 idosos para cada 100 crianças, em 2000 essa relação passou para quase 30 idosos para cada 100 crianças 1 . A população idosa apresenta níveis de morbidade maiores que o da população em geral, com maior consumo de medicamentos e procura por serviços de saúde. Os medicamentos estão entre as intervenções mais utilizadas e de grande valor no tratamento de doenças nesse grupo etário, aumentando a sobrevida e melhorando a qualidade de vida 2 . Estudos revelam que a prevalência de uso de ao menos um medicamento é superior a $90 \%$, considerando períodos curtos como 7 ou 14 dias anteriores às entrevistas domiciliares ou realizadas nos serviços de saúde 3,4,5,6. Prevalências um pouco menores (82-86\%) foram verificados em estudos realizados em municípios com menos de 70 mil habitantes 7,8 .

Os estudos de utilização de medicamentos, dedicados a fornecer estimativas de padrão de uso de medicamentos em diferentes populações, 
raramente incluem dados da população rural, provavelmente pelas limitações relacionadas com os recursos e o tempo necessários ao deslocamento das equipes de campo para as áreas rurais. Idosos moradores em áreas rurais podem apresentar um padrão de uso de medicamentos diferente da população urbana, em termos qualitativos e quantitativos. A indisponibilidade de serviços de saúde próximos ao local de residência, incluindo unidades de atenção básica à saúde e farmácias, ou o acesso reduzido a esses serviços disponíveis em outros locais, por dificuldades de transporte, podem constituir fatores importantes para o menor consumo de medicamentos. Restrições financeiras e isolamento social do morador da área rural também podem contribuir para o menor acesso aos serviços de saúde e, consequentemente, ao consumo de medicamentos 9,10

Estudos comparando fatores relacionados ao uso de medicamentos em populações de municípios de pequeno porte e que incluam os moradores das áreas rurais são escassos 9,11,12. Não foram localizados estudos realizados no Brasil com o propósito de avaliar a influência do local de moradia no uso de medicamentos, utilizando amostras de base populacional.

A identificação de padrões de uso de medicamentos pelos moradores da área rural, comparado aos padrões urbanos, poderá contribuir para identificar necessidades de saúde em populações rurais e, assim, estabelecer prioridades para tais populações no que diz respeito ao acesso e à utilização adequada de medicamentos.

O objetivo deste estudo é avaliar o uso de medicamentos entre idosos residentes em áreas urbanas e rurais de município no Sul do Brasil.

\section{Métodos}

O presente estudo está baseado nos dados coletados no período de julho a dezembro de 2004, no Município de Carlos Barbosa, localizado no Rio Grande do Sul, o Estado mais ao sul do Brasil. O município, que está localizado a 104km ao norte da capital, possuía 20.519 habitantes no ano de 2000 , e desses, $10,6 \%$ tinham idade igual ou superior a 60 anos, e 79,3\% moravam na área urbana, de acordo com o Censo Demográfico de 2000 (Instituto Brasileiro de Geografia e Estatística. http://www.ibge.gov.br/home/estatistica/popu lacao/default_censo_2000.shtm).A esperança de vida ao nascer era de 75,44 em 2000, superior à média do Rio Grande do Sul $(72,05)$ e do Brasil $(70,44) 13$. O Índice de Desenvolvimento Humano municipal (IDH-M) era de 0,858, acima dos índices estaduais $(0,814)$ e nacional $(0,766) 14$.

\section{População e amostra}

Os critérios de elegibilidade foram: ter 60 anos ou mais, viver de maneira independente e ser saudável. Foram definidas pessoas saudáveis aquelas cujo estado físico, médico e mental as permitissem locomover-se e participar de um exame de saúde bucal conduzido em uma clínica, uma vez que o presente estudo é parte de um estudo maior a respeito da percepção de saúde bucal 15 .

Com base num censo de idosos residentes nas áreas urbanas e rurais realizado em 2003 pela Secretaria Municipal de Assistência Social e Habitação de Carlos Barbosa, foi gerado um banco de dados dos idosos que totalizavam, na época do estudo, 2.167 indivíduos com idade igual ou superior a 60 anos. Vinte e dois idosos vivendo em casa de repouso foram considerados inelegíveis. Os idosos elegíveis (2.145) foram sorteados por meio de uma tabela de números aleatórios. $\mathrm{O}$ tamanho da amostra sorteado (983) foi definido para atender ao objetivo do estudo principal. Dos potenciais participantes, um indivíduo mudouse para outro município e nove morreram antes da coleta dos dados. Não foi possível incluir 113 indivíduos após três ou mais tentativas de contato por telefone e carta. De um total de 983 idosos contatados, 13 estavam acamados, um hospitalizado e 97 recusaram participar da pesquisa, resultando em 872 participantes.

\section{Aferição}

A coleta de dados ocorreu por meio de entrevistas presenciais realizadas nas residências dos participantes ou em clubes da comunidade. Antes do início das entrevistas, os participantes eram informados a respeito dos objetivos do estudo e solicitados a assinar termo de consentimento informado. Um pesquisador treinado lia cada questão em voz alta e registrava as respostas em um questionário padronizado. O tempo médio de duração da entrevista foi de 60 minutos.

Variáveis sociodemográficas e variáveis relacionadas à saúde obtidas pelas entrevistas foram categorizadas para os propósitos das análises estatísticas. A idade foi categorizada em: 60-69 anos, 70-79 anos e $\geq 80$ anos; a raça autorreferida em: branca e não branca; a situação conjugal em: com companheiro e sem companheiro; a escolaridade em: até 4 anos e mais de 4 anos; a localização da residência em: urbana e rural; a renda familiar em $\leq \mathrm{R} \$ 260,00$ e $\mathrm{R}$ \$ 261,00-R \$ 3.500,00; e o número de doenças crônicas em: nenhuma, uma, e duas ou mais. O ponto de corte utilizado para a renda foi a mediana, correspondente ao salário mínimo em vigor na época da coleta 
dos dados ( $\mathrm{R} \$ 260,00$ ). A qualidade de vida foi avaliada em quatro domínios: físico, psicológico, social e ambiental, utilizando-se do instrumento WHOQOL-BREF, traduzido e validado para o português 16. As pontuações foram categorizadas pelas medianas de cada domínio, assumindo que os idosos com valores iguais ou inferiores à mediana apresentavam pior qualidade de vida que os idosos com valores acima da mediana, para cada domínio avaliado.

A definição de zona urbana ou rural seguiu os critérios estabelecidos em lei municipal, a qual define zona urbana aquela que apresenta melhoramentos em pelo menos dois dos seguintes itens: meio-fio ou calçamento, abastecimento de água, sistema de esgotos sanitários, rede de iluminação pública, escola ou posto de saúde próximos. A zona rural é definida como área utilizada em atividades agropecuárias, agroindustriais, extrativismo, silvicultura e conservação ambiental. De posse da lista dos bairros centrais caracterizados como zona urbana e das localidades rurais e do endereço dos idosos, disponibilizados pela prefeitura previamente ao sorteio da amostra, classificamos os idosos como moradores da zona urbana ou rural do município.

As variáveis de desfecho foram: uso de medicamentos e polifarmácia. O uso de medicamentos foi autorreferido, por meio de uma questão fechada seguida por uma questão aberta. A fim de reduzir potencial viés recordatório e de auxiliar na identificação dos medicamentos, solicitava-se aos idosos que apresentassem as embalagens de todos os medicamentos de uso contínuo.

O uso de medicamentos foi definido como o uso contínuo de medicamentos na época da entrevista. O uso contínuo foi definido como o uso diário de medicamentos por orientação médica. Não foi investigado o uso eventual de medicamentos. A polifarmácia foi definida mediante a contagem dos medicamentos usados concomitantemente, utilizando o ponto de corte de cinco ou mais medicamentos, de acordo com outros autores 17,18 .

Os medicamentos foram classificados em categorias terapêuticas pelo sistema de classificação Anatomical Therapeutic Chemical (ATC) da Organização Mundial da Saúde 19. Por esse sistema, as substâncias ativas são divididas em diferentes grupos de acordo com o órgão ou sistema em que atuam, e suas propriedades terapêuticas, farmacológicas e químicas. Os medicamentos são classificados em grupos de cinco níveis diferentes. No primeiro nível, são classificados dentro de 14 grupos principais, com subgrupos farmacológicos/terapêuticos (segundo nível). O terceiro e quarto níveis são subgrupos químicos / farmacológicos/terapêuticos e o quinto nível é a substância química. No presente estudo, classificamos os medicamentos até o segundo nível.

\section{Análises estatísticas}

As análises estatísticas foram realizadas utilizando o programa estatístico SPSS versão 18.0 (SPSS Inc., Chicago, Estados Unidos). Foram realizadas análises descritivas para as variáveis sociodemográficas (sexo, raça autorreferida, situação conjugal, idade, escolaridade e renda familiar), doenças crônicas, qualidade de vida e variáveis sobre o uso de medicamentos, de acordo com a localização da residência (área urbana ou rural). Foi utilizado o teste do qui-quadrado para comparação de proporções e o teste de tendência linear para variáveis ordinais.

A associação entre local de moradia e os desfechos uso de medicamentos e polifarmácia foram estimados por intermédio da razão de prevalência (RP). Para as estimativas das RP ajustadas para outras variáveis preditoras, foram desenvolvidos modelos por regressão de Poisson com ajuste robusto da variância. Na primeira etapa da construção do modelo, as variáveis foram analisadas individualmente. Foram testados fatores sociodemográficos (sexo, raça autorreferida, situação conjugal, idade, escolaridade e renda familiar), número de doenças crônicas e qualidade de vida. As variáveis que apresentaram significância estatística, definida como $\mathrm{p}<0,20$, nas análises univariadas, foram selecionadas para a segunda etapa, em que todas as variáveis foram introduzidas no modelo. As variáveis que apresentaram $\mathrm{p}>0,05$, nessa etapa, foram retiradas, uma a uma do modelo, mantendo-se fixa a variável preditora principal (local de moradia) e idade, independente do valor de $\mathrm{p}$.

\section{Aspectos éticos}

Este estudo foi aprovado pelo Comitê de Ética em Pesquisa da Faculdade de Odontologia da Universidade Estadual de Campinas (UNICAMP - protocolo no. 055/2004), por estar adequado ética e metodologicamente e de acordo com a Resolução $n^{\circ}$. 196/1996 e complementares do Conselho Nacional de Saúde. Os participantes assinaram termo de consentimento informado antes de participar da pesquisa.

\section{Resultados}

A amostra de 872 idosos não apresentou diferenças em relação à idade, sexo, situação conjugal e local de moradia quando comparada com a população de idosos com 60 anos ou mais de 
Carlos Barbosa 20. Para a presente análise, foram excluídos 61 idosos sobre os quais não dispomos de informações sobre os medicamentos usados, totalizando 811 indivíduos analisados (93\% da amostra inicial). A amostra analisada não apresentou diferenças em relação à amostra excluída quanto às variáveis sexo, cor da pele, situação conjugal, renda familiar e número de doenças crônicas. Os idosos excluídos dessa análise moravam mais na área rural (44) do que os analisados (409) e possuíam aproximadamente três anos a mais, em média $(71,03 \pm 7,13)$ do que os analisados $(68,29 \pm 6,63)$.

As principais características sociodemográficas relacionadas à saúde da amostra analisada são apresentadas na Tabela 1, segundo o local de moradia. A maioria dos idosos era mulher, com idade inferior a 70 anos, de cor de pele branca, morando com companheiro, com baixa escolaridade, renda igual ou inferior a 260 reais e portadores de duas ou mais doenças crônicas. A predominância dessas características manteve-se independentemente do local de moradia, com exceção da renda familiar. Moradores da área urbana apresentaram maior renda que os moradores da área rural. Em relação à qualidade de vida, verificamos maior percentual de moradores na área rural com maior qualidade de vida, para os domínios psicológico, social e ambiental, que os moradores na área urbana.

O uso contínuo de medicamentos foi referido por 586 idosos $(72,3 \%)$, com uma média de 2,1 medicamentos utilizados diariamente e máximo de 13 medicamentos. A polifarmácia foi verificada em $13,9 \%$ da amostra. Os moradores da área urbana usaram mais medicamentos que os da área rural (Tabela 2). A Tabela 2 ainda informa os medicamentos mais utilizados pelos idosos, classificados em subgrupos farmacológicos/terapêuticos.

A Tabela 3 apresenta as prevalências e RP de uso de medicamentos, de acordo com características sociodemográficas, para todos os idosos e por local de moradia. A prevalência de uso de medicamentos foi maior nas mulheres, com mais idade, raça autorreferida branca, morando sem companheiro, com maior escolaridade, renda superior a 260 Reais, com maior número de doenças e pior qualidade de vida. Os moradores na área urbana apresentaram prevalência de uso de medicamentos superior às dos moradores na área rural, para todas as características analisadas.

As prevalências e RP de polifarmácia, de acordo com as características sociodemográficas e local de moradia dos idosos, são apresentadas na Tabela 4. A distribuição das prevalências de polifarmácia foi semelhante à observada para uso de medicamentos, para as características analisadas, incluindo local de moradia.

A associação positiva entre área urbana e uso de medicamentos verificada na análise bruta (RP = 1,22; IC95\%: 1,11-1,32) manteve-se após análise ajustada, (RP = 1,10; IC95\%: 1,021,20). A associação positiva entre área urbana e polifarmácia verificada na análise bruta $(\mathrm{RP}=$ 2,36; IC95\%: 1,62-3,50) manteve-se na análise ajustada (RP = 1,83; IC95\%: 1,27-2,65). A Tabela 5 mostra os resultados dos modelos finais ajustados para potenciais confundidores, para uso de medicamentos e polifarmácia.

\section{Discussão}

Este estudo avaliou a prevalência de uso de medicamentos entre idosos de Carlos Barbosa e comparou as características sociodemográficas, econômicas e de saúde associadas ao uso de medicamentos e polifarmácia, segundo o local de moradia.

O uso contínuo de medicamentos verificado neste estudo $(72,3 \%)$ foi inferior aos resultados encontrados em outros trabalhos realizados no país 3,5,21, que verificaram prevalências superiores a $80 \%$. Estudos realizados nos Estados Unidos com idosos apresentando limitações funcionais 6 , e na Alemanha, com idosos com 70 anos ou mais 4, apontam para prevalências próximas a 100\%. Tais discrepâncias podem ser decorrentes não apenas das diferenças de consumo entre as populações estudadas, mas também dos critérios de seleção da amostra e se a amostra é de base populacional ou não. É possível que nossa amostra tenha sido composta por indivíduos mais saudáveis que a dos estudos citados, se considerarmos que os idosos de Carlos Barbosa referiram menor número de doenças crônicas 5,14, eram mais jovens 4 e não apresentavam limitações funcionais importantes ${ }^{6}$. Além desses fatores, características demográficas e econômicas dos municípios onde os idosos residem parecem estar associados ao menor uso de medicamentos. Entre as menores prevalências de uso de medicamentos verificadas em estudos brasileiros, encontram-se aquelas verificadas em municípios de pequeno porte localizados no interior (82 e 86,1\%) 7,8, e em áreas de baixo nível socioeconômico $(60,7 \%) 22$.

Na presente análise, a prevalência de uso foi menor na área rural em comparação à área urbana, diferença que se manteve após controle para fatores de confusão (sexo, escolaridade, renda familiar, número de doenças crônicas e domínio físico). Não localizamos na literatura outro estudo com idosos brasileiros morando na área rural, 
Características sociodemográficas, econômicas e de saúde dos idosos residentes na área urbana e rural. Carlos Barbosa, Rio Grande do Sul, Brasil, 2004 ( $n=811)$ *.

\begin{tabular}{|c|c|c|c|c|c|c|c|}
\hline \multirow[t]{2}{*}{ Característica } & \multicolumn{2}{|c|}{ Área urbana } & \multicolumn{2}{|c|}{ Área rural } & \multicolumn{2}{|c|}{ Total } & \multirow{2}{*}{$\begin{array}{c}\text { Valor de } \\
\qquad p^{\star \star}\end{array}$} \\
\hline & n & $\%$ & $\mathbf{n}$ & $\%$ & $\mathbf{n}$ & $\%$ & \\
\hline Sexo & & & & & & & 0,91 \\
\hline Feminino & 262 & 65,2 & 264 & 64,5 & 526 & 64,8 & \\
\hline Masculino & 140 & 34,8 & 145 & 35,5 & 285 & 35,1 & \\
\hline Idade (anos) & & & & & & & 0,619 \\
\hline $60-69$ & 240 & 59,7 & 247 & 61,1 & 487 & 60,4 & \\
\hline $70-79$ & 130 & 32,3 & 128 & 31,7 & 258 & 32,0 & \\
\hline$\geq 80$ & 32 & 8,0 & 29 & 7,2 & 61 & 7,6 & \\
\hline Raça autorreferida & & & & & & & 0,02 \\
\hline Branca & 385 & 96,2 & 396 & 99,0 & 781 & 97,6 & \\
\hline Não branca & 15 & 3,8 & 4 & 1,0 & 19 & 2,4 & \\
\hline Situação conjugal & & & & & & & 0,024 \\
\hline Com companheiro & 273 & 68,1 & 308 & 75,5 & 581 & 71,8 & \\
\hline Sem companheiro & 128 & 31,9 & 100 & 24,5 & 228 & 28,2 & \\
\hline Escolaridade (anos) & & & & & & & 0,013 \\
\hline Até 4 & 274 & 68,3 & 310 & 76,4 & 584 & 72,4 & \\
\hline Mais de 4 & 127 & 31,7 & 96 & 23,6 & 223 & 27,6 & \\
\hline Renda familiar & & & & & & & $<0,001$ \\
\hline$\leq \mathrm{R} \$ 260,00$ & 189 & 47,0 & 274 & 67,2 & 463 & 57,2 & \\
\hline $\mathrm{R} \$ 261,00-\mathrm{R} \$ 3.500,00$ & 213 & 53,0 & 134 & 32,8 & 347 & 42,8 & \\
\hline Número de doenças crônicas & & & & & & & 0,001 \\
\hline 0 & 66 & 16,5 & 89 & 22,1 & 155 & 19,3 & \\
\hline 1 & 84 & 20,9 & 115 & 28,6 & 199 & 24,8 & \\
\hline$\geq 2$ & 251 & 62,6 & 198 & 49,3 & 449 & 55,9 & \\
\hline \multicolumn{8}{|c|}{ Qualidade de vida (WHOQOL-BREF) } \\
\hline Domínio físico & & & & & & & 0,374 \\
\hline$\leq$ Mediana & 206 & 51,6 & 219 & 55,0 & 425 & 53,3 & \\
\hline$>$ Mediana & 193 & 48,4 & 179 & 45,0 & 372 & 46,7 & \\
\hline Domínio psicológico & & & & & & & 0,148 \\
\hline$\leq$ Mediana & 216 & 53,9 & 193 & 48,5 & 409 & 51,2 & \\
\hline$>$ Mediana & 185 & 46,1 & 205 & 51,5 & 390 & 48,8 & \\
\hline Domínio social & & & & & & & $<0,001$ \\
\hline$\leq$ Mediana & 234 & 58,4 & 176 & 44,0 & 410 & 51,2 & \\
\hline > Mediana & 167 & 41,6 & 224 & 56,0 & 391 & 48,8 & \\
\hline Domínio ambiental & & & & & & & 0,018 \\
\hline$\leq$ Mediana & 232 & 57,9 & 196 & 49,2 & 428 & 53,6 & \\
\hline$>$ Mediana & 169 & 42,1 & 202 & 50,8 & 371 & 46,4 & \\
\hline
\end{tabular}

* A variação no total de participantes nas categorias resulta de dados ausentes relacionados a cada variável em questão;

** Teste do qui-quadrado.

paracomparação dos achados. Estudos realizados nos Estados Unidos e Canadá apresentam dados discordantes 11. Estudo conduzido por Hanlon et al. 23, na Carolina do Norte (Estados Unidos), aponta a área urbana como fator independente para uso de medicamentos, ao passo que outros autores não verificaram qualquer associação em amostras de idosos residentes no Texas (Estados Unidos) 24 e em Manitoba (Canadá) 9.

A prevalência de polifarmácia entre os idosos de Carlos Barbosa foi de 13,9\%, inferior ao encontrado no Rio de Janeiro (32,7\%) 21 e em Porto Alegre (27\%) 3 , mas próximo do encontrado em Fortaleza $(5,4$ a $13 \%){ }^{22}$. A polifarmácia foi mais 
Uso de medicamentos de acordo com o local de moradia. Carlos Barbosa, Rio Grande do Sul, Brasil, 2004 ( $n=811$ ).

\begin{tabular}{|c|c|c|c|c|c|c|c|}
\hline \multirow[t]{2}{*}{ Característica } & \multicolumn{2}{|c|}{$\begin{array}{l}\text { Área urbana } \\
\text { (n = 402) }\end{array}$} & \multicolumn{2}{|c|}{$\begin{array}{l}\text { Área rural } \\
(n=409)\end{array}$} & \multicolumn{2}{|c|}{ Total } & \multirow[t]{2}{*}{$\begin{array}{l}\text { Valor } \\
\text { de p }\end{array}$} \\
\hline & $\mathbf{n}$ & $\%$ & $\mathrm{n}$ & $\%$ & $\mathbf{n}$ & $\%$ & \\
\hline Usou medicamentos & 319 & 79,4 & 267 & 63,5 & 586 & 72,3 & $<0,050$ * \\
\hline Número médio de medicamentos/dia & 2,40 & $(0-13)$ & 1,80 & $(0-10)$ & 2,11 & $(0-13)$ & $<0,050 * \star$ \\
\hline Polifarmácia (5 ou mais medicamentos) & 79 & 19,7 & 34 & 8,3 & 113 & 13,9 & $<0,050$ * \\
\hline \multicolumn{8}{|l|}{ Medicamentos mais utilizados } \\
\hline C03 - diuréticos & 110 & 27,4 & 70 & 17,1 & 180 & 22,2 & $<0,050$ * \\
\hline C02 - anti-hipertensivos & 79 & 19,7 & 68 & 16,6 & 147 & 18,1 & 0,304 * \\
\hline N06A - antidepressivos & 68 & 16,9 & 48 & 11,7 & 116 & 14,3 & 0,045 * \\
\hline B01 - antitrombóticos & 57 & 14,2 & 46 & 11,2 & 103 & 12,7 & $0,251 *$ \\
\hline C01D - vasodilatadores usados em doenças cardíacas & 54 & 13,4 & 45 & 11,0 & 99 & 12,2 & 0,342 * \\
\hline C10 - antilipêmicos & 42 & 10,4 & 31 & 7,6 & 73 & 9,0 & 0,192 * \\
\hline
\end{tabular}

* Teste qui-quadrado;

** Teste t.

prevalente entre os idosos residentes na área urbana de Carlos Barbosa. A associação mantevese após ajuste por idade, número de doenças crônicas e domínio físico. A prevalência mais alta de polifarmácia na área urbana pode estar relacionada à maior facilidade de acesso aos serviços de saúde, o que leva a maior diagnóstico de doenças crônicas e, consequentemente, maior número de medicamentos prescritos. Não foram localizados estudos comparando diferenças na prevalência de polifarmácia entre idosos moradores na área urbana e rural. Em uma revisão de estudos que avaliaram a intensidade de uso, os achados de pesquisas realizadas na Finlândia e nos estados da Pensilvânia e Carolina do Norte, nos Estados Unidos, foram inconsistentes 11.

Nos modelos finais ajustados, o domínio físico do WHOQOL permaneceu como variável independente associada ao uso de medicamentos e à polifarmácia. Esse domínio avalia a qualidade de vida do idoso relacionada com o funcionamento físico. Podemos esperar que indivíduos mais doentes e que usem mais medicamentos tenham uma percepção pior sobre sua função física. Entre os aspectos avaliados nesse domínio, está a percepção do indivíduo sobre a dependência de medicamentos ou de outros tratamentos. No entanto, dada a natureza transversal do estudo, não podemos descartar a possibilidade de causalidade reversa, para essa associação.

Poucos estudos têm investigado diferenças em relação ao padrão de acesso e utilização de serviços de saúde entre populações rurais e urbanas, incluindo acesso a medicamentos 11,24 . No contexto brasileiro, uma análise sobre o acesso a serviços de saúde, com base em dados da Pesquisa Nacional por Amostra de Domicílios de 2003 (PNAD), revela que a utilização de serviços de saúde pelos idosos residentes na área rural foi menor do que nos idosos urbanos, todavia não apresenta dados específicos sobre o uso de medicamentos 25. Em Carlos Barbosa, o Programa Saúde da Família ainda não foi implementado, e a carência de profissionais médicos nas unidades de saúde localizadas nos cinco distritos que compõem a área rural, além da ausência de farmácia ou posto de medicamentos, podem contribuir para o uso de medicamentos em menor escala. Deslocar-se do local de moradia para realizar consultas médicas ou adquirir medicamentos pode ser particularmente difícil para os idosos que dependem exclusivamente de familiares para o deslocamento, ou para aqueles residentes em localidades mais distantes, desprovidas de transporte coletivo regular, ou que residem em locais com topografia mais acidentada, dificultando a caminhada até a parada de ônibus. Aspectos relacionados ao trabalho rural também podem interferir no uso dos medicamentos. Trabalhadores rurais que permanecem o dia inteiro na lavoura podem desistir de utilizar um ou mais medicamentos com mais frequência do que os urbanos, se o uso for dificultado pelas condições próprias do trabalho rural (medicamentos que necessitem ser administrados várias vezes ao dia ou que necessitem de técnicas e aparatos especiais para a administração, como é o caso da insulina, por exemplo). 
Prevalência e razão de prevalências (RP) bruta para o uso de medicamentos em idosos, por área de moradia e por variáveis sociodemográficas, econômicas e de saúde. Carlos Barbosa, Rio Grande do Sul, Brasil, 2004.

\begin{tabular}{|c|c|c|c|c|c|c|c|c|c|}
\hline & \multicolumn{3}{|c|}{ Área urbana $(n=402)$} & \multicolumn{3}{|c|}{ Área rural $(n=409)$} & \multicolumn{3}{|c|}{ Total $(n=811)$} \\
\hline & $\%$ & RP (IC95\%) & Valor de $p$ * & $\%$ & RP (IC95\%) & Valor de p * & $\%$ & RP (IC95\%) & Valor de p * \\
\hline \multicolumn{10}{|l|}{ Sexo } \\
\hline Feminino & 85,5 & $1,26(1,1-1,43)$ & $<0,001$ & 73,1 & $1,43(1,20-1,71)$ & $<0,001$ & 79,3 & $1,34(1,20-1,49)$ & $<0,001$ \\
\hline Masculino & 67,9 & 1,00 & & 51,0 & 1,00 & & 59,3 & 1,00 & \\
\hline \multicolumn{10}{|l|}{ Idade (anos) } \\
\hline $60-69$ & 77,1 & 1,00 & 0,244 & 62,3 & 1,00 & 0,115 & 69,6 & 1,00 & 0,045 \\
\hline $70-79$ & 83,1 & $1,08(0,97-1,20)$ & & 69,5 & $1,12(0,96-1,30)$ & & 76,4 & $1,10(1,00-1,20)$ & \\
\hline$\geq 80$ & 81,3 & $1,05(0,88-1,26)$ & & 72,4 & $1,16(0,91-1,48)$ & & 77,0 & $1,11(0,95-1,28)$ & \\
\hline \multicolumn{10}{|l|}{ Raça } \\
\hline Branca & 79,7 & $1,20(0,83-1,72)$ & 0,368 & 65,9 & $2,64(0,48-14,41)$ & 0,236 & 72,7 & $1,26(0,85-1,85)$ & 0,240 \\
\hline Não branca & 66,7 & 1,00 & & 25,0 & 1,00 & & 57,9 & 1,00 & \\
\hline \multicolumn{10}{|l|}{ Situação conjugal } \\
\hline Com companheiro & 76,9 & 1,00 & 0,076 & 63,0 & 1,00 & 0,088 & 69,5 & 1,00 & 0,004 \\
\hline Sem companheiro & 85,2 & $1,11(1,00-1,22)$ & & 73,0 & $1,16(1,00-1,34)$ & & 79,8 & $1,15(1,05-1,25)$ & \\
\hline \multicolumn{10}{|l|}{ Escolaridade (anos) } \\
\hline Até 4 & 77,4 & 1,00 & 0,205 & 63,9 & 1,00 & 0,346 & 70,2 & 1,00 & 0,045 \\
\hline$\geq 5$ & 83,5 & $1,08(0,98-1,19)$ & & 69,8 & $1,09(0,93-1,28)$ & & 77,6 & $1,11(1,01-1,21)$ & \\
\hline \multicolumn{10}{|l|}{ Renda familiar } \\
\hline$\leq \mathrm{R} \$ 260,00$ & 76,7 & 1,00 & 0,269 & 63,5 & 1,00 & 0,360 & 68,9 & 1,00 & 0,018 \\
\hline$>\mathrm{R} \$ 260,00$ & 81,7 & $1,06(0,96-1,18)$ & & 68,7 & $1,08(0,93-1,25)$ & & 76,7 & $1,11(1,02-1,21)$ & \\
\hline \multicolumn{10}{|l|}{ Número de doenças } \\
\hline 0 & 30,3 & 1,00 & $<0,001$ & 21,3 & 1,00 & $<0,001$ & 25,2 & 1,00 & \\
\hline 1 & 78,6 & $2,59(1,77-3,80)$ & & 62,6 & $2,93(1,92-4,48)$ & & 69,3 & $2,76(2,07-3,67)$ & $<0,001$ \\
\hline$\geq 2$ & 92,4 & $3,05(2,11-4,41)$ & & 86,9 & $4,07(2,72-6,09)$ & & 90,0 & $3,58(2,72-4,70)$ & $<0,001$ \\
\hline \multicolumn{10}{|l|}{ Domínio físico } \\
\hline$\leq$ Mediana & 93,2 & $1,42(1,27-1,58)$ & $<0,001$ & 76,3 & $1,45(1,24-1,70)$ & $<0,001$ & 84,5 & $1,42(1,30-1,56)$ & $<0,001$ \\
\hline$>$ Mediana & 65,8 & 1,00 & & 52,5 & 1,00 & & 59,4 & 1,00 & \\
\hline \multicolumn{10}{|l|}{ Domínio psicológico } \\
\hline$\leq$ Mediana & 84,7 & $1,15(1,04-1,28)$ & 0,008 & 69,9 & $1,14(0,99-1,31)$ & 0,094 & 77,8 & $1,16(1,06-1,26)$ & 0,001 \\
\hline$>$ Mediana & 73,5 & 1,00 & & 61,4 & 1,00 & & 67,2 & 1,00 & \\
\hline \multicolumn{10}{|l|}{ Domínio social } \\
\hline$\leq$ Mediana & 82,9 & $1,11(1,00-1,23)$ & 0,065 & 65,3 & $0,99(0,86-1,14)$ & 0,963 & 75,4 & $1,08(0,99-1,18)$ & 0,093 \\
\hline$>$ Mediana & 74,9 & 1,00 & & 66,1 & 1,00 & & 69,8 & 1,00 & \\
\hline \multicolumn{10}{|l|}{ Domínio ambiental } \\
\hline$\leq$ Mediana & 80,6 & $1,03(0,93-1,14)$ & 0,626 & 63,3 & $0,93(0,81-1,08)$ & 0,395 & 72,7 & $1,00(0,92-1,09)$ & 1,000 \\
\hline$>$ Mediana & 78,1 & 1,00 & & 67,8 & 1,00 & & 72,5 & 1,00 & \\
\hline
\end{tabular}

IC95\%: intervalo de 95\% de confiança; RP: razão de prevalência.

* Teste qui-quadrado.

Os medicamentos com ação sobre o aparelho cardiovascular (categoria C pela classificação ATC) foram os mais utilizados, em concordância com estudos prévios 3,5,7,8,22. Uma vez que doenças cardiovasculares estão entre as principais causas de morbimortalidade entre idosos, esses medicamentos têm sido amplamente prescritos. Destaca-se, entretanto, o elevado uso de antide- pressivos, sobretudo entre os idosos residentes na área urbana.

O presente estudo apresenta algumas limitações. O viés de memória não pode ser descartado quando a informação sobre o uso dos medicamentos restringe-se ao relato do respondente. Procuramos minimizar o problema pela solicitação das embalagens dos medicamentos em uso 
Prevalência e razão de prevalências bruta para polifarmácia em idosos, por área de moradia e por variáveis sociodemográficas, econômicas e de saúde. Carlos Barbosa, Rio Grande do Sul, Brasil, 2004.

\begin{tabular}{|c|c|c|c|c|c|c|c|c|c|}
\hline \multirow[t]{2}{*}{ Variável } & \multicolumn{3}{|c|}{$\begin{array}{l}\text { Área urbana } \\
(n=402)\end{array}$} & \multicolumn{3}{|c|}{$\begin{array}{l}\text { Área rural } \\
(n=409)\end{array}$} & \multicolumn{3}{|c|}{$\begin{array}{c}\text { Total } \\
(\mathrm{n}=811)\end{array}$} \\
\hline & $\%$ & RP (IC95\%) & Valor de $p$ * & $\%$ & RP (IC95\%) & Valor de $p$ * & $\%$ & RP (IC95\%) & Valor de $p$ * \\
\hline \multicolumn{10}{|l|}{ Sexo } \\
\hline Feminino & 21,4 & $1,30(0,84-2,02)$ & 0,290 & 10,2 & $2,12(0,95-4,74)$ & 0,088 & 15,8 & $1,50(1,01-2,22)$ & 0,050 \\
\hline Masculino & 16,4 & 1,00 & & 4,8 & 1,00 & & 10,5 & 1,00 & \\
\hline \multicolumn{10}{|l|}{ Idade (anos) } \\
\hline $60-69$ & 13,3 & 1,00 & 0,001 & 7,3 & 1,00 & 0,070 & 10,3 & 1,00 & $<0,001$ \\
\hline $70-79$ & 30,0 & $2,25(1,48-3,41)$ & & 7,8 & $1,07(0,51-2,25)$ & & 19,0 & $1,85(1,29-2,66)$ & \\
\hline$\geq 80$ & 25,0 & $1,88(0,95-3,71)$ & & 20,7 & $2,84(1,23-6,58)$ & & 23,0 & $2,24(1,32-3,80)$ & \\
\hline \multicolumn{10}{|l|}{ Raça autorreferida } \\
\hline Branca & 19,7 & $1,48(0,40-5,46)$ & 0,745 & 8,6 & & 1,000 & 14,1 & $1,34(0,36-5,02)$ & 1,000 \\
\hline Não branca & 13,3 & 1,00 & & 0,0 & 1,00 & & 10,5 & 1,00 & \\
\hline \multicolumn{10}{|l|}{ Situação conjugal } \\
\hline Com companheiro & 20,1 & 1,00 & 0,847 & 6,8 & 1,00 & 0,083 & 13,1 & 1,00 & 0,294 \\
\hline Sem companheiro & 18,8 & $0,93(0,60-1,43)$ & & 13,0 & $1,91(0,99-3,67)$ & & 16,2 & $1,24(0,86-1,78)$ & \\
\hline \multicolumn{10}{|l|}{ Escolaridade (anos) } \\
\hline Até 4 & 20,1 & 1,00 & 0,888 & 8,1 & 1,00 & 1,000 & 13,7 & 1,00 & 0,900 \\
\hline$\geq 5$ & 18,9 & $0,94(0,61-1,45)$ & & 8,3 & $1,03(0,48-2,22)$ & & 14,3 & $1,05(0,72-1,53)$ & \\
\hline \multicolumn{10}{|l|}{ Renda familiar } \\
\hline$\leq \mathrm{R} \$ 260,00$ & 20,1 & 1,00 & 0,928 & 5,8 & 1,00 & 0,016 & 11,7 & 1,00 & 0,039 \\
\hline$>\mathrm{R} \$ 260,00$ & 19,2 & $0,96(0,64-1,42)$ & & 13,4 & $2,30(1,21-4,37)$ & & 17,0 & $1,46(1,04-2,05)$ & \\
\hline \multicolumn{10}{|l|}{ Número de doenças } \\
\hline 0 & 3,0 & 1,00 & $<0,001$ & 1,1 & 1,00 & $<0,001$ & 1,9 & 1,00 & $<0,001$ \\
\hline 1 & 7,1 & $2,36(0,49-11,30)$ & & 1,7 & $1,55(0,14-16,80)$ & & 4,0 & $2,08(0,56-7,70)$ & \\
\hline$\geq 2$ & 28,3 & $9,33(2,35-37,06)$ & & 15,7 & $13,931,93-100,48$ & & 22,7 & $11,74(3,78-36,46)$ & \\
\hline \multicolumn{10}{|l|}{ Domínio físico } \\
\hline$\leq$ Mediana & 31,6 & $4,35(2,53-7,49)$ & $<0,001$ & 13,2 & $4,74(1,87-12,00)$ & $<0,001$ & 22,1 & $4,33(2,70-6,95)$ & $<0,001$ \\
\hline > Mediana & 7,3 & 1,00 & & 2,8 & 1,00 & & 5,1 & 1,00 & \\
\hline \multicolumn{10}{|l|}{ Domínio psicológico } \\
\hline$\leq$ Mediana & 25,5 & $1,96(1,27-3,04)$ & 0,003 & 8,8 & $1,06(0,56-2,02)$ & 0,996 & 17,6 & $1,67(1,17-2,39)$ & 0,004 \\
\hline$>$ Mediana & 13,0 & 1,00 & & 8,3 & 1,00 & & 10,5 & 1,00 & \\
\hline \multicolumn{10}{|l|}{ Domínio social } \\
\hline$\leq$ Mediana & 23,9 & $1,74(1,12-2,71)$ & 0,017 & 6,8 & $0,69(0,35-1,36)$ & 0,374 & 16,6 & $1,44(1,02-2,05)$ & 0,050 \\
\hline$>$ Mediana & 13,8 & 1,00 & & 9,8 & 1,00 & & 11,5 & 1,00 & \\
\hline \multicolumn{10}{|l|}{ Domínio ambiental } \\
\hline$\leq$ Mediana & 23,3 & $1,57(1,02-2,42)$ & 0,048 & 6,1 & $0,56(0,29-1,10)$ & 0,128 & 15,4 & $1,22(0,86-1,72)$ & 0,312 \\
\hline$>$ Mediana & 14,8 & 1,00 & & 10,9 & 1,00 & & 12,7 & 1,00 & \\
\hline
\end{tabular}

IC95\%: intervalo de 95\% de confiança; RP: razão de prevalência.

* Teste qui-quadrado.

contínuo. Não avaliamos o uso eventual de medicamentos, fato que pode explicar o baixo percentual de medicamentos analgésicos, ao contrário dos achados de outros estudos 3,6,7. Não consultamos registros médico-ambulatoriais ou hospitalares dos participantes.
A generalização dos resultados deste estudo deve ser feita com cautela, pelas diferenças que a população investigada pode apresentar em relação a outros municípios brasileiros. O Município de Carlos Barbosa apresenta indicadores sociais, econômicos e de saúde superiores às médias do estado e do Brasil, além de características 
Razão de prevalência ajustada do uso de medicamentos e polifarmácia em idosos. Carlos Barbosa, Rio Grande do Sul, Brasil, 2004.

\begin{tabular}{|c|c|c|}
\hline & RP (IC95\%) & Valor de $p$ * \\
\hline \multicolumn{3}{|l|}{ Uso de medicamentos } \\
\hline \multicolumn{3}{|l|}{ Área } \\
\hline Urbana & $1,10(1,02-1,20)$ & 0,014 \\
\hline Rural & 1,00 & \\
\hline \multicolumn{3}{|l|}{ Sexo } \\
\hline Feminino & $1,18(1,07-1,30)$ & 0,001 \\
\hline Masculino & 1,00 & \\
\hline \multicolumn{3}{|l|}{ Escolaridade (anos) } \\
\hline Até 4 & 1,00 & 0,026 \\
\hline$\geq 5$ & $1,09(1,01-1,18)$ & \\
\hline \multicolumn{3}{|l|}{ Renda familiar } \\
\hline$\leq \mathrm{R} \$ 260,00$ & 1,00 & 0,013 \\
\hline$>\mathrm{R} \$ 260,00$ & $1,10(1,02-1,19)$ & \\
\hline Número de doenças crônicas & $1,15(1,12-1,18)$ & $<0,001$ \\
\hline \multicolumn{3}{|l|}{ Domínio físico } \\
\hline$\leq$ Mediana & $1,21(1,10-1,32)$ & $<0,001$ \\
\hline > Mediana & 1,00 & \\
\hline \multicolumn{3}{|l|}{ Polifarmácia } \\
\hline \multicolumn{3}{|l|}{ Área } \\
\hline Urbana & $1,83(1,27-2,65)$ & 0,001 \\
\hline Rural & 1,00 & \\
\hline Idade (anos) & $1,03(1,01-1,05)$ & 0,012 \\
\hline Número de doenças crônicas & $1,42(1,30-1,55)$ & $<0,001$ \\
\hline \multicolumn{3}{|l|}{ Domínio físico } \\
\hline$\leq$ Mediana & $2,68(1,63-4,41)$ & $<0,001$ \\
\hline > Mediana & 1,00 & \\
\hline
\end{tabular}

IC95\%: intervalo de 95\% de confiança; RP: razão de prevalência.

* Teste qui-quadrado.

culturais fortemente associadas aos antepassados italianos e alemães responsáveis pela colonização do município e região, que podem influenciar no estilo de vida e nos cuidados com a saúde. Além disso, variáveis importantes não foram testadas nas análises multivariadas, tais como plano de saúde, número de consultas médicas e de hospitalizações no último ano e meios de transporte utilizados.

A avaliação do uso de medicamentos pela amostra representativa de uma população idosa do sul do Brasil, incluindo idosos residentes na área rural, vem contribuir para os avanços no conhecimento nessa área. Conforme apontado por Coelho Filho et al. 22, grande parte dos estudos farmacoepidemiológicos realizados no Brasil apresenta como fator limitante a seleção de amostras de conveniência, pouco representativas da população geriátrica. Amostras rurais ra- ramente são incluídas pelas dificuldades logísticas associadas, especialmente aquelas relacionadas ao tempo e recursos financeiros necessários ao deslocamento das equipes de campo. Além disso, efeitos de sazonalidade no uso de determinados medicamentos, como aqueles utilizados em problemas respiratórios foram minimizados, pois o estudo estendeu-se entre os meses de julho e dezembro.

Os resultados deste trabalho mostram que o uso de medicamentos e a polifarmácia é maior entre idosos moradores da área urbana de Carlos Barbosa. O papel dos hábitos de vida, crenças e valores dos idosos moradores em diferentes áreas na procura por serviços de saúde e utilização de medicamentos precisa ser investigado, bem como fatores relacionados ao sistema de saúde e condições de acesso aos serviços e aos medicamentos. 


\section{Resumo}

O objetivo foi verificar a prevalência de uso de medicamentos e de polifarmácia entre idosos de Carlos Barbosa, Rio Grande do Sul, Brasil, e comparar as características sociodemográficas e de saúde associadas ao uso, segundo o local de moradia. Foi avaliada uma amostra aleatória de 811 idosos com 60 anos ou mais, moradores na área urbana ou rural. Dados sociodemográficos, doenças crônicas, qualidade de vida e medicamentos autorreferidos foram coletados em entrevistas presenciais. Associação entre local de moradia e uso de medicamentos ou polifarmácia, ajustada para potenciais confundidores, foi avaliada por regressão de Poisson com ajuste robusto da variância. A prevalência de uso de medicamentos e de polifarmácia foi maior entre os idosos urbanos. Morar na área urbana apresentou associação positiva e independente com uso de medicamentos $(R P=1,10$; IC95\%: 1,02-1,20) $e$ polifarmácia (RP = 1,83; IC95\%: 1,27-2,65). Morar na área urbana está associado à maior prevalência de uso de medicamentos e de polifarmácia entre idosos.

Idoso; Uso de Medicamentos; Acesso aos Serviços de Saúde; Saúde da População Rural

\section{Referências}

1. Instituto Brasileiro de Geografia e Estatística. Perfil dos idosos responsáveis pelos domicílios no Brasil 2000. Rio de Janeiro: Instituto Brasileiro de Geografia e Estatística; 2002.

2. Murray MD, Callahan CM. Improving medication use for older adults: an integrated research agenda. Ann Intern Med 2003; 139(5 Pt 2):425-9.

3. Flores LM, Mengue SS. Uso de medicamentos por idosos em região do sul do Brasil. Rev Saúde Pública 2005 ; 39:924-9.

4. Junius-Walker U, Theile G, Hummers-Pradier E. Prevalence and predictors of polypharmacy among older primary care patients in Germany. Fam Pract 2007; 24:14-9.

5. Ribeiro AQ, Rozenfeld S, Klein CH, César CC, Acurcio FA. Inquérito sobre uso de medicamentos por idosos aposentados, Belo Horizonte, MG. Rev Saúde Pública 2008; 42:724-32.

6. Crentsil V, Ricks MO, Xue QL, Fried LP. A pharmacoepidemiologic study of community-dwelling, disabled older women: factors associated with medication use. Am J Geriatr Pharmacother 2010; 8:215-24.

\section{Colaboradores}

T. S. Dal Pizzol e E. S. Pons participaram do planejamento e análises dos dados, redação, revisão do manuscrito e aprovação da versão final. F. N. Hugo e J. B. Hilgert participaram do planejamento do estudo, realizaram a coleta de dados e inserção dos dados, revisão do manuscrito e aprovação da versão final. M. C. Bozzetti e M. L. R. Sousa participaram do planejamento do estudo, revisão do manuscrito e aprovação da versão final.
7. Loyola Filho AI, Uchoa E, Firmo JOA, Lima-Costa MF. Estudo de base populacional sobre o consumo de medicamentos entre idosos: Projeto Bambuí. Cad Saúde Pública 2005; 21:545-53.

8. Flores VB, Benvegnú LA. Perfil de utilização de medicamentos em idosos da zona urbana de Santa Rosa, Rio Grande do Sul, Brasil. Cad Saúde Pública 2008; 24:1439-46.

9. Carrie AG, Grymonpre RE, Blandford AA. Impact of residence on prevalence and intensity of prescription drug use among older adults. Ann Pharmacother 2006; 40:1932-8.

10. Goins RT, Williams KA, Carter MW, Spencer M, Solovieva T. Perceived barriers to health care access among rural older adults: a qualitative study. J Rural Health 2005; 21:206-13.

11. Grymonpre RE, Hawranik PG. Rural residence and prescription medication use by community-dwelling older adults: a review of the literature. J Rural Health 2008; 24:203-9. 
12. Cesar JA, Oliveira-Filho JA, Bess G, Cegielka R, Machado J, Gonçalves TS, et al. Perfil dos idosos residentes em dois municípios pobres das regiões Norte e Nordeste do Brasil: resultados de estudo transversal de base populacional. Cad Saúde Pública 2008; 24:1835-45.

13. Fundação de Economia e Estatística Siegfried Emanuel Heuser. Resumo estatístico RS. http:// www.fee.tche.br/sitefee/pt/content/resumo/pg municipios_detalhe.php?municipio=Carlos + Barbosa (acessado em 13/Dez/2010).

14. Programa das Nações Unidas para o Desenvolvimento. Atlas do desenvolvimento humano no Brasil, 2003. http://www.pnud.org.br/atlas/ranking/ IDH-M\%2091\%2000\%20Ranking\%20decrescen te\%20(pelos\%20dados\%20de\%202000).htm (acessado em 13/Dez/2010).

15. Hugo FN, Hilgert JB, Sousa ML, Cury JA. Oral status and its association with general quality of life in older independent-living south-Brazilians. Community Dent Oral Epidemiol 2009; 37:231-40.

16. Fleck MP, Louzada S, Xavier M, Chachamovich E, Vieira G, Santos L, et al. Aplicação da versão em português do instrumento abreviado de avaliação da qualidade de vida "WHOQOL-bref". Rev Saúde Pública 2000; 34:178-83.

17. Hanlon JT, Schmader KE, Ruby CM, Weinberger M. Suboptimal prescribing in older inpatients and outpatients. J Am Geriatr Soc 2001; 49:200-9.

18. Klarin I, Fastbom J, Wimo A. A population-based study of drug use in the very old living in a rural district of Sweden, with focus on cardiovascular drug consumption: comparison with an urban cohort. Pharmacoepidemiol Drug Saf 2003; 12:669-78.
19. Anatomical Therapeutic Chemical. Classification Index with defined daily doses (DDDs). World Health Organization; 2010. http://www.whocc.no/ atc_ddd_index/(acessado em 13/Dez/2010).

20. Hilgert JB, Hugo FN, Sousa ML, Bozzetti MC. Oral status and its association with obesity in Southern Brazilian older people. Gerodontology 2009; 26:4652.

21. Rozenfeld S, Fonseca MJ, Acurcio FA. Drug utilization and polypharmacy among the elderly: a survey in Rio de Janeiro City, Brazil. Rev Panam Salud Pública 2008; 23:34-43.

22. Coelho Filho JM, Marcopito LF, Castelo A. Perfil de utilização de medicamentos por idosos em área urbana do Nordeste do Brasil. Rev Saúde Pública 2004; 38:557-64.

23. Hanlon JT, Landerman LR, Wall WE, Horner RD, Fillenbaum GG, Dawson DV, et al. Is medication use by community-dwelling elderly people influenced by cognitive function? Age Ageing 1996; 25:190-6.

24. Xu KT, Smith SR, Borders TF. Access to prescription drugs among noninstitutionalized elderly people in west Texas. Am J Health Syst Pharm 2003; 60:675-82

25. Travassos C, Viacava F. Acesso e uso de serviços de saúde em idosos residentes em áreas rurais, Brasil, 1998 e 2003. Cad Saúde Pública 2007; 23:2490-502.

Recebido em 09/Mar/2011

Versão final reapresentada em 31/Ago/2011

Aprovado em 15/Set/2011 